\title{
Cosmic positron and antiproton constraints on the gauge-Higgs Dark Matter
}

\author{
Kingman Cheung ${ }^{1,2,3}$, Jeonghyeon Song ${ }^{1}$, and Po-Yan Tseng ${ }^{2}$ \\ ${ }^{1}$ Division of Quantum Phases \& Devices, \\ School of Physics, Konkuk university, Seoul 143-701, Korea \\ ${ }^{2}$ Department of Physics, National Tsing Hua University, Hsinchu 300, Taiwan \\ ${ }^{3}$ Physics Division, National Center for Theoretical Sciences, Hsinchu 300, Taiwan
}

(Dated: November 3, 2018)

\begin{abstract}
We calculate the cosmic ray positron and antiproton spectra of a gauge-Higgs dark matter candidate in a warped five-dimensional $S O(5) \times U(1)$ gauge-Higgs unification model. The stability of the gauge-Higgs boson is guaranteed by the $H$ parity under which only the Higgs boson is odd at low energy. The 4-point vertices of $H H W^{+} W^{-}$and $H H Z Z$, allowed by $H$ parity conservation, have the same magnitude as in the standard model, which yields efficient annihilation rate for $m_{H}>m_{W}$. The most dominant annihilation channel is $H H \rightarrow W^{+} W^{-}$followed by the subsequent decays of the $W$ bosons into positrons or quarks, which undergo fragmentation into antiproton. Comparing with the observed positron and antiproton spectra with the PAMALA and Fermi/LAT, we found that the Higgs boson mass cannot be larger than $90 \mathrm{GeV}$, in order not to overrun the observations. Together with the constraint on not overclosing the Universe, the valid range of the dark matter mass is restricted to $70-90 \mathrm{GeV}$.
\end{abstract}




\section{INTRODUCTION}

Since Lee and Weinberg put bound on a stable neutrino mass from the relic density of the Universe in 1977 [1], various cosmological and astrophysical observations have inspired and also constrained many theoretical models in particle physics. In particular the very precise measurement of the cosmic microwave background radiation in the Wilkinson Microwave Anisotropy Probe (WMAP) experiment established the presence of cold dark matter (CDM) in our Universe [2]. These data are urging new physics (NP) beyond the standard model (SM) to provide the stable CDM particle over cosmological time scale.

Recently, a number of high energy cosmic ray experiments suggested the possibility of indirect detections of CDM annihilation in the galactic halo. The Payload for Antimatter Matter Exploration and Light-nuclei Astrophysics (PAMELA) collaboration reported the excess of positrons in its energy spectrum of $10-100 \mathrm{GeV}$ over the expected high energy cosmic ray interaction with the interstellar medium [3]. More mysterious result was that the antiproton data, on the other hand, seem to be consistent with the expected spectrum from the collisions between high-energy protons in cosmic rays and the nuclei of hydrogen and helium atoms in the interstellar medium [4]. In addition, the Fermi/LAT [5] and the HESS [6] collaborations also reported a smooth but harder electron-flux spectrum than the expected background at 100-1000 GeV.

In the literature there have been great efforts to explain these anomalous data by the annihilation of CDM particles in NP models, such as the lightest supersymmetric particle [7], the Kaluza-Klein particle [8], and other CDM candidates [9]. It was shown that many CDM particle candidates such as in supersymmetric (SUSY) models and the universal extra dimensional (UED) model require a large boost factor to explain the observed signal. Even though the inhomogeneity of CDM [10] or the Sommerfeld mechanism [11] can help, the val-

ues of these boost factors are limited. Some other NP models [9], e.g., a new long range force in the dark sector [12], can explain the PAMELA data without extreme boost factor.

In this paper we adopt a different approach to these astrophysical data, i.e., using them to constrain a NP theory, instead of explaining them to support a theory. The positron excess could be explained by astrophysical sources like pulsars [13] or supernova remnants [14]. Historically the astrophysical observations have played the role of a strong constraint on a NP model. For instance, the very precise WMAP measurement of the relic density eliminates 
a large portion of the parameter space of the constrained minimal supersymmetric SM to avoid overclosing the Universe [15].

We will study the positron and antiproton energy spectra from CDM annihilation in a recently proposed $S O(5) \times U(1)$ gauge-Higgs unification model based on a warped fivedimensional (5D) spacetime [16-18]. Here the dark matter is nothing but the Higgs boson [19], which is a part of the fifth component of a gauge boson field in the model. The $H$ parity, under which only the Higgs boson has odd parity at low energy, is preserved from the gauge structure of the theory. Triple vertices such as $W W H, Z Z H$, and $\bar{f} f H$ (here $f$ is a SM fermion) vanish to all orders in perturbation theory [19, 20], thus the Higgs boson is stable and becomes a dark matter candidate. Instead, the 4-point vertices of $H H W^{+} W^{-}$, $H H Z Z$, and $H H f \bar{f}$ are allowed by the $H$ parity conservation. The Higgs boson can be thermally produced in the early Universe via $W W, Z Z, f \bar{f} \rightarrow H H$, of which the rate is highly predictive with essentially one free parameter, the Higgs boson mass $m_{H}$.

In Ref. [19], it was shown that the Higgs boson mass needs to be at $70 \mathrm{GeV}$ in order to explain the WMAP data. When $m_{H}<70 \mathrm{GeV}$, the total annihilation rate is very small because the kinematically allowed $2 \rightarrow 2$ processes into light fermions are suppressed by small Yukawa couplings. The corresponding relic density becomes too big, which overcloses the Universe. If $m_{H}>70 \mathrm{GeV}$, the cross section of $H H \rightarrow W W^{(*)}$ becomes very large, since the magnitude of $H H W W$ coupling is the same as in the SM. The corresponding relic density is too low. Nevertheless, this is not ruled out. It is possible that the dark matter can be produced nonthermally from, e.g., other quasi-stable Kaluza-Klein states or other topological objects. In this work, we assume that the thermal source is not the only source of relic dark matter, and so Higgs boson mass can be larger than $70 \mathrm{GeV}$. The present upper bound on the Higgs mass comes from the unitarity requirement, which limits $m_{H}<\mathcal{O}(1)$ $\mathrm{TeV}[21]$.

We note that this large Higgs mass yields too efficient annihilation into $W^{+} W^{-}$and possibly $Z Z$, which leads to potentially large positron and antiproton signals. Using the observed positron and antiproton spectra in the PAMELA and Fermi/LAT experiments, we can set the upper limit on the Higgs boson mass. On the other hand, the collider signal for this model is way too small for detection [22].

In this work, we study the positron and antiproton spectra from annihilation of the gauge-Higgs dark matter in the halo. We use the cosmic ray propagation code Galprop [23] 
to calculate the propagation of the positron and antiproton from the halo to the Earth, and compare with the spectra measured by PAMELA [3, 4]. The most dominant annihilation channel for $m_{H} \geq 70 \mathrm{GeV}$ is $H H \rightarrow W^{+} W^{-}$, followed by the subsequent decays of the $W$ bosons into positrons or quarks. The quarks undergo fragmentation into antiproton. Here we do not attempt to explain the anomaly observed by PAMELA. Instead we use them as the constraints on the model: the resulting spectra obtained from the CDM annihilation should not exceed the ones measured by PAMELA.

It is well known that the process $H H \rightarrow W^{+} W^{-}$grows with the center-of-mass (c.m.) energy $\sqrt{s}$. Naively, the longitudinal polarization of the $W$ boson behaves like $p^{\mu} / m_{W}$ when $\sqrt{s} \gg m_{W}$. Therefore, we expect the scattering amplitude squared grows as $s^{2} / m_{W}^{4}$ before any UV physics comes in to unitarize the theory. ${ }^{1}$ For the CDM annihilation in the halo where $v \approx 10^{-3}$, the c.m. energy is just roughly $2 m_{H}$. Thus, we expect the annihilation rate will grow with the Higgs boson mass, as rapidly as $m_{H}^{2} / m_{W}^{2}$, so long as $m_{H}<1 \mathrm{TeV}$. We will show that when the Higgs boson mass is $90 \mathrm{GeV}$ or above, the resulting positron spectrum is already well above that measured by PAMELA. Similar conclusion holds true for the antiproton spectrum. We can, therefore, conclude that the Higgs boson mass cannot be larger than about $90 \mathrm{GeV}$ in this gauge-Higgs model. This is the main result of our work.

The organization of the paper is as follows. In the next section, we briefly review the model and describe the effective interactions used in this calculation. We give details about the calculation of the positron spectrum and antiproton spectrum in Secs. III and IV] respectively. We also perform the comparison with the measured spectra. We conclude in Sec.V.

\section{THE EFFECTIVE INTERACTIONS IN THE $S O(5) \times U(1)$ GAUGE-HIGGS MODEL}

The model under consideration is a $S O(5) \times U(1)$ gauge-Higgs unification model in the 5D Randall-Sundrum warped space [16]. The Higgs boson is the fluctuation mode of the Aharonov-Bohm phase $\hat{\theta}_{H}$ along the fifth dimension [24], i.e., $\hat{\theta}_{H}=\theta_{H}+H(x) / f_{H}$. The

\footnotetext{
${ }^{1}$ In this gauge-Higgs model based on five-dimensional spacetime, it is the Kaluza-Klein states (of order $\mathrm{TeV}$ ) of the gauge bosons which unitarize the scattering amplitude.
} 
four-dimensional effective Lagrangian of the Higgs boson is

$$
\mathcal{L}=V_{\text {eff }}\left(\hat{\theta}_{H}\right)-m_{W}^{2}\left(\hat{\theta}_{H}\right) W_{\mu}^{+} W^{-\mu}-\frac{1}{2} m_{Z}^{2}\left(\hat{\theta}_{H}\right) Z_{\mu} Z^{\mu}-\sum_{f} m_{f}\left(\hat{\theta}_{H}\right) \bar{\psi}_{f} \psi_{f},
$$

where the mass functions are

$$
m_{W}\left(\hat{\theta}_{H}\right)=\frac{1}{2} g f_{H} \sin \hat{\theta}_{H}, \quad m_{Z}\left(\hat{\theta}_{H}\right)=\frac{1}{2} g_{Z} f_{H} \sin \hat{\theta}_{H}, \quad m_{f}\left(\hat{\theta}_{H}\right)=y_{f} f_{H} \sin \hat{\theta}_{H} .
$$

Here $g$ is the weak gauge coupling and $g_{Z}=g / \cos \theta_{W}$.

The effective potential $V_{\text {eff }}\left(\hat{\theta}_{H}\right)$ of the Higgs boson is generated at one loop level. It is finite and cutoff independent. As shown in Ref. [16], the large contribution of 5D top quark field changes the global minimum of $V_{\text {eff }}\left(\hat{\theta}_{H}\right)$ into $\hat{\theta}_{H}= \pm \pi / 2$ : the $W, Z$ gauge bosons as well as the SM fermions acquire their masses (see Eq.(2) ) and thus the electroweak symmetry is broken dynamically.

In this model the global minimum at $\hat{\theta}_{H}= \pm \pi / 2$ dynamically generates a new $H$ parity, under which the Higgs boson has odd parity while all the other SM particles have even parity. This $H$-parity prohibits triple vertices of the Higgs boson with the SM particles, such as $H W^{+} W^{-}, H Z Z$ and $H f \bar{f}$ : it preserves the stability of the Higgs boson so that the Higgs boson can be a CDM candidate.

At low energy this model has two parameters, $f_{H}$ and $m_{H}$. The parameter $f_{H}$ is determined by the observed $m_{W}$ and $m_{Z}$, i.e., $f_{H} \approx 246 \mathrm{GeV}$. The value of $m_{H}$ is, in principle, determined if the whole matter content in the model is fixed in detail. Without a priori knowledge of UV physics, we treat $m_{H}$ as a free parameter.

Because of the absence of the triple vertices of the Higgs boson with the SM particles, we do not have significant constraint on $m_{H}$ from collider physics phenomenology. Instead the observed relic density of CDM in the WMAP experiment can provide a meaningful one [19]. If the Higgs boson mass becomes heavier, their annihilation into $W^{+} W^{-}$and possibly into $Z Z$ are kinematically accessible, leading to smaller relic density of the Universe. On the other hand, lighter $m_{H}$ yields too small annihilation cross section, which is excluded as overclosing the Universe. The Higgs boson mass $m_{H}=70 \mathrm{GeV}$ can explain the observed relic density. We employ the WMAP data as not overclosing the universe: the Higgs boson mass can be heavier than $70 \mathrm{GeV}$.

The effective interactions used in this work are

$$
\mathcal{L}=\frac{1}{8} g_{Z}^{2} H^{2} Z_{\mu} Z^{\mu}+\frac{1}{4} g^{2} H^{2} W_{\mu}^{+} W^{-\mu}+\sum_{f} \frac{m_{f}}{2 f_{H}^{2}} H^{2} \bar{\psi}_{f} \psi_{f} .
$$


Note that the $H H W^{+} W^{-}$and $H H Z Z$ vertices have the same couplings with the SM ones except for the opposite sign. Since the annihilation cross section of $H H \rightarrow W^{+} W^{-}, Z Z$ increases with the c.m. energy $\sqrt{s}$ (or $m_{H}$ when the relative velocity of two $H$ 's is very small), heavier Higgs boson could leave too much excess of positrons and antiprotons from $W$ or $Z$ decays.

\section{POSITRON SPECTRUM}

The dominant process for the cosmic ray positrons from the Higgs boson annihilation is the leptonic decay of the $W^{+}$boson:

$$
H H \rightarrow W^{+} W^{-} \rightarrow e^{+}+\nu_{e}+X .
$$

The next dominant process is $H H \rightarrow Z Z \rightarrow e^{+}+X$. Positrons can also come from the hadrons, which are the fragmentation products of the quarks from $W$ decays. These positrons are much softer than those coming directly from the $W$ boson decay. We shall ignore these soft positrons. There are also other processes $H H \rightarrow f \bar{f}$ (for $f=b, c$ ) to produce positrons in the subsequent decays of the fragmentation products, but they are certainly subleading because of the small Yukawa couplings compared with the gauge coupling.

A back-of-envelope calculation shows that the annihilation rate is given by

$$
\begin{aligned}
\langle\sigma v\rangle_{H H \rightarrow W W} & \equiv \sigma\left(H H \rightarrow W^{+} W^{-}\right)\left(2 \beta_{H}\right)=\frac{g^{4} \beta_{W}}{32 \pi s}\left(3-\frac{s}{m_{W}^{2}}+\frac{s^{2}}{4 m_{W}^{4}}\right), \\
\langle\sigma v\rangle_{H H \rightarrow Z Z} & \equiv \sigma(H H \rightarrow Z Z)\left(2 \beta_{H}\right)=\frac{g_{z}^{4} \beta_{Z}}{64 \pi s}\left(3-\frac{s}{m_{Z}^{2}}+\frac{s^{2}}{4 m_{Z}^{4}}\right),
\end{aligned}
$$

where $2 \beta_{H}=2 \sqrt{1-4 m_{H}^{2} / s}$ is the relative velocity of the two non-relativistic incoming Higgs bosons in their c.m. frame, and $\beta_{W, Z}=\sqrt{1-4 m_{W, Z}^{2} / s}$. It is easy to see that the annihilation rate grows as $s / m_{W, Z}^{2}$.

The positron flux observed at the Earth is given by

$$
\Phi_{e^{+}}(E)=\frac{v_{e^{+}}}{4 \pi} f_{e^{+}}(E),
$$

with $v_{e^{+}}$is close to the velocity of light $c$. The function $f_{e^{+}}(E)$ satisfies the diffusion equation of

$$
\frac{\partial f}{\partial t}-K(E) \nabla^{2} f-\frac{\partial}{\partial E}(b(E) f)=Q
$$


where the diffusion coefficient is $K(E)=K_{0}(E / \mathrm{GeV})^{\delta}$ and the energy loss coefficient is $b(E)=E^{2} /\left(\mathrm{GeV} \times \tau_{E}\right)$ with $\tau_{E}=10^{16}$ sec. The source term $Q$ due to the annihilation is

$$
Q_{\mathrm{ann}}=\eta\left(\frac{\rho_{\mathrm{CDM}}}{M_{\mathrm{CDM}}}\right)^{2} \sum\langle\sigma v\rangle_{e^{+}} \frac{d N_{e^{+}}}{d E_{e^{+}}}
$$

where $\eta=1 / 2$ for identical scalar DM particle in the initial state. The summation is over all possible channels that can produce positrons in the final state, and $d N_{e^{+}} / d E_{e^{+}}$denotes the spectrum of the positron energy per annihilation in that particular channel.

In our analysis, the source term is given by

$$
Q_{\mathrm{ann}}=\frac{1}{2}\left(\frac{\rho_{\mathrm{CDM}}}{M_{\mathrm{CDM}}}\right)^{2}\left[\langle\sigma v\rangle_{H H \rightarrow W W} \frac{d N_{e^{+}}^{W W}}{d E_{e^{+}}}+\langle\sigma v\rangle_{H H \rightarrow Z Z} \frac{d N_{e^{+}}^{Z Z}}{d E_{e^{+}}}\right]
$$

where the normalizations of $N_{e^{+}}^{W W}$ and $N_{e^{+}}^{Z Z}$ are

$$
\int \frac{d N_{e^{+}}^{W W}}{d x} d x=B\left(W^{+} \rightarrow e^{+} \nu_{e}\right), \quad \int \frac{d N_{e^{+}}^{Z Z}}{d x} d x=2 \times B\left(Z \rightarrow e^{+} e^{-}\right) .
$$

We first calculate the energy spectrum of the positron in the $W^{+}$or $Z$ rest frame, then boost it to the c.m. frame of the $H H$ system. In the calculation we include all the off-shell effects of the $W^{+}$and $W^{-}$bosons and in both $Z$ bosons. We then put the source term into Galprop [23] to solve the diffusion equation.

Note that the contribution from $H H \rightarrow Z Z$ channel is subleading in our calculation. It only accounts for about $1 \%$ and $4 \%$ of the contribution from $H H \rightarrow W^{+} W^{-}$channel for $m_{H}=82$ and $90 \mathrm{GeV}$, respectively. For $m_{H}=100 \mathrm{GeV}$, the $Z Z$ channel contribution is as large as $20 \%$ of the $W W$ one. It is easy to understand: for $m_{H}$ below $90 \mathrm{GeV}$ the $Z Z$ channel is below the production threshold and thus one of the $Z$ bosons has to be off-shell, while at $m_{H}=100 \mathrm{GeV}$ both $Z$ bosons are already on shell.

Figure 1 shows the positron spectra for $m_{H}=70,82,90$, and $100 \mathrm{GeV}$ with the measured spectrum of PAMELA [3]. For $m_{H}=70 \mathrm{GeV}$, the gauge-Higgs CDM annihilation cannot explain the rising feature in the positron spectrum. The annihilation rate is too low since the $W^{+} W^{-}$channel is not kinematically open yet. When the Higgs boson mass is above $m_{W}$, the annihilation rate increases quickly and can explain part of the rise-up in the spectrum, as shown by the $m_{H}=82 \mathrm{GeV}$ curve. Yet, it still cannot explain the two highest energy points because the mass of the Higgs boson is not heavy enough. Once the Higgs boson mass rises to $90-100 \mathrm{GeV}$, it passes through the second highest energy point, but the 


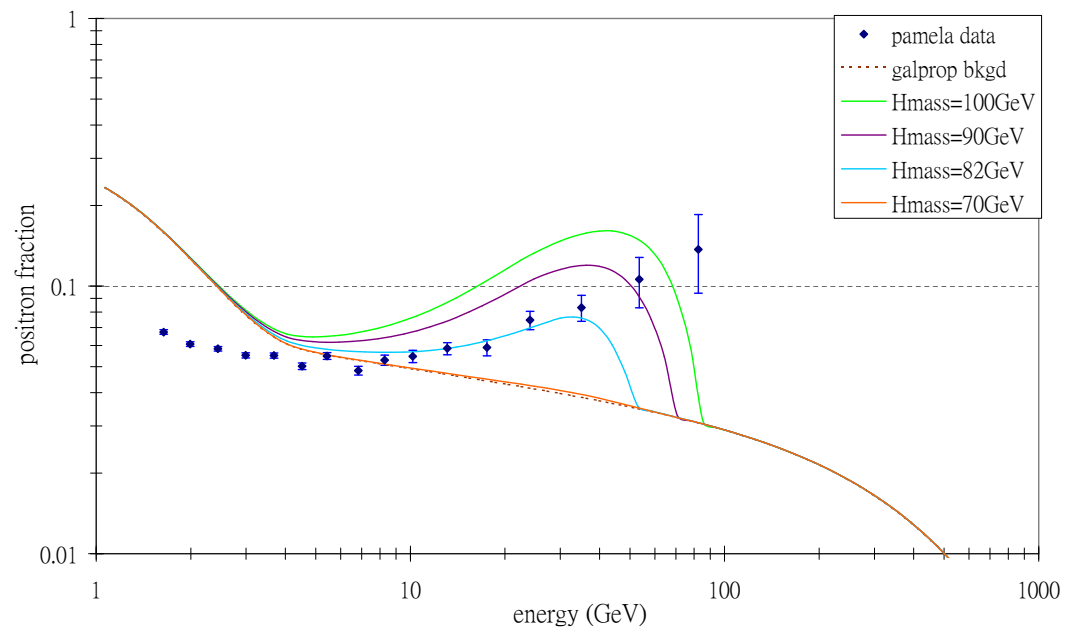

FIG. 1: The positron-fraction spectrum predicted for the gauge-Higgs dark matter model for $m_{H}=70,82,90,100 \mathrm{GeV}$, assuming that it accounts for all the dark matter of the Universe. The PAMELA data are also shown.

annihilation rate increases far more than the lower part of the spectrum allowed. Just by visual checking, $m_{H}$ larger than $90 \mathrm{GeV}$ is easily ruled out by the measured spectrum.

We also show the total electron and positron flux in Fig. 2, Our results are compared with the measurement of the cosmic ray $e^{+}+e^{-}$spectra from $20 \mathrm{GeV}$ to $1 \mathrm{TeV}$ with the Fermi LAT [5], and from $340 \mathrm{GeV}$ with the HESS [6]. These cosmic ray electron spectra are distributed over very high energy up to $1 \mathrm{TeV}$. Unless the CDM mass is very heavy about $800-1000 \mathrm{GeV}$, the whole energy spectrum cannot be explained. Instead of explaining high energy spectrum, we focus on the region of $20 \mathrm{GeV} \lesssim E \lesssim 100 \mathrm{GeV}$. The case of $m_{H}=82 \mathrm{GeV}$ is marginally allowed by the data while the $m_{H}=90 \mathrm{GeV}$ case already outruns the observation. 


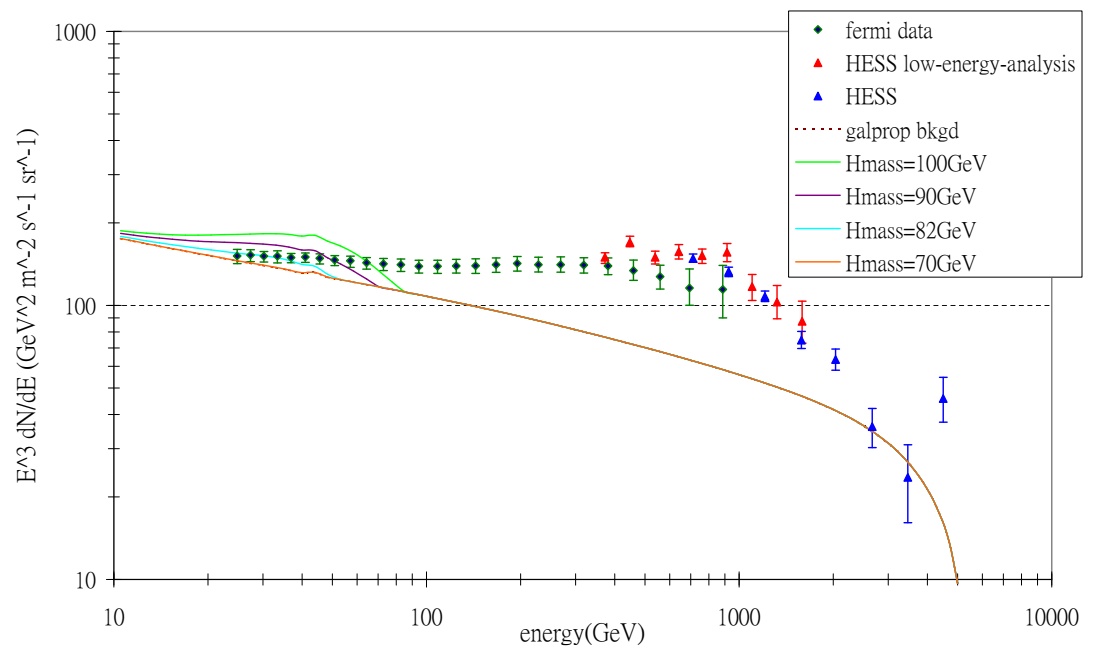

FIG. 2: Total flux of electron and positron predicted for the gauge-Higgs dark matter model for $m_{H}=70,82,90,100 \mathrm{GeV}$. Data from Fermi/LAT and HESS are shown.

\section{ANTIPROTON SPECTRUM}

Similar to the treatment for positron flux, the antiproton flux can be obtained by solving the diffusion equation with an appropriate source term for the input antiproton spectrum:

$$
Q_{\mathrm{ann}}=\eta\left(\frac{\rho_{\mathrm{dm}}}{M_{\mathrm{dm}}}\right)^{2} \sum\langle\sigma v\rangle_{\bar{p}} \frac{d N_{\bar{p}}}{d T_{\bar{p}}},
$$

where $\eta=1 / 2$, and $T_{\bar{p}}$ is the kinetic energy of the antiproton which is conventionally used instead of the total energy. We solve the diffusion equation using Galprop [23].

In our case, the dominant contribution to the cosmic ray antiproton production comes from

$$
H H \rightarrow W^{+} W^{-} \rightarrow\left(q \bar{q}^{\prime}\right)\left(q \bar{q}^{\prime}\right) \rightarrow \bar{p}+X
$$

followed by $H H \rightarrow Z Z \rightarrow(q \bar{q})\left(q^{\prime} \bar{q}^{\prime}\right) \rightarrow \bar{p}+X$. In the last step, we adopt a publicly available code [25] to calculate the fragmentation function $D_{q \rightarrow h}(z)$ for any quark $q$ into hadrons $h$, e.g., $p, \bar{p}, \pi$. The fragmentation function is then convoluted with energy spectrum $d N / d E$ of the light quark to obtain the energy spectrum of the antiproton $d N / d E_{\bar{p}}$. The next contribution comes from $H H \rightarrow b \bar{b} \rightarrow \bar{p}+X$. Since the annihilation is smaller by two 


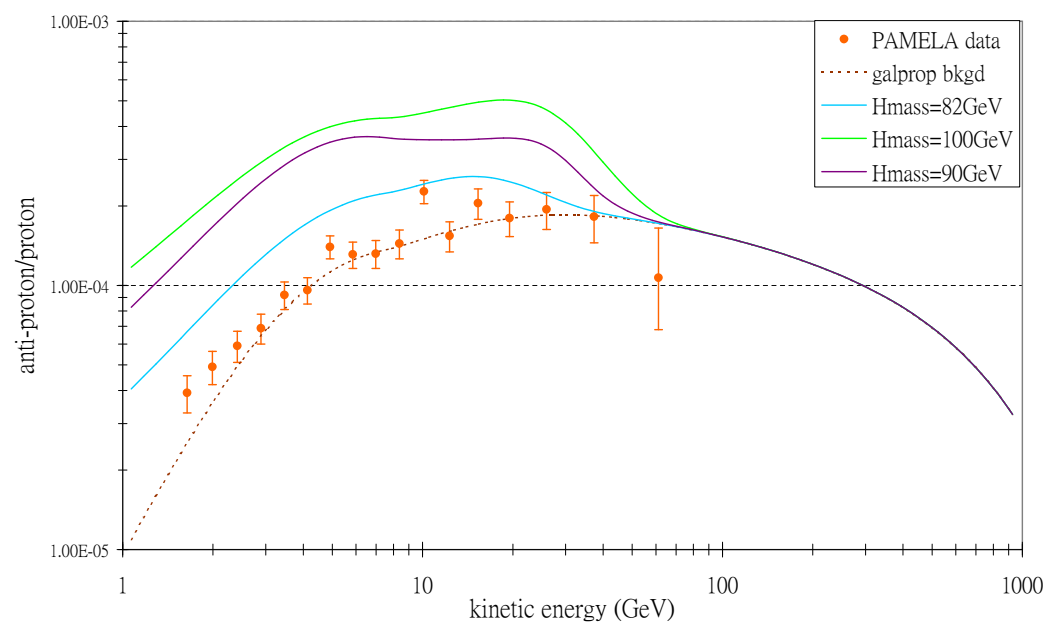

FIG. 3: The fraction of the antiproton $\bar{p} /(p+\bar{p})$ versus the kinetic energy of the antiproton predicted for the gauge-Higgs dark matter model for $m_{H}=82,90,100 \mathrm{GeV}$. The antiproton data of PAMELA are also shown.

orders of magnitude, we ignore this and the other subleading contributions. The source term $d N / d T_{\bar{p}}$ is then implemented into Galprop to calculate the propagation from the halo to the Earth.

The resulting antiproton fraction as a function of its energy is shown in Fig. 3, Three theoretical curves are for $m_{H}=82,90,100 \mathrm{GeV}$, compared with the observed PAMELA data. Note that in this figure we only include the leading contribution from $H H \rightarrow W^{+} W^{-}$ because the next subleading contribution only accounts for less than $5 \%$ of the leading one for $m_{H} \leq 90 \mathrm{GeV}$. We can see that the $m_{H}=82$ (just above the $W W$ threshold) curve is barely consistent with the data. On the other hand, the $m_{H}=90$ and $100 \mathrm{GeV}$ curves are obviously over the data.

Since the theoretical background curve contains relatively high uncertainties due to different models used in Galprop, we do not perform any confidence-level exclusion analysis. Instead, we only use a visual inspection. Very conservatively, we conclude that the $m_{H}=90$ $\mathrm{GeV}$ or above is excluded by the antiproton spectrum. A similar conclusion can be drawn from the positron spectrum. Overall, $m_{H}=90 \mathrm{GeV}$ or above is strongly disfavored by the 
data

\section{CONCLUSIONS}

In the $S O(5) \times U(1)$ gauge-Higgs unification model based on the Randall-Sundrum warped spacetime, the Higgs boson is a cold dark matter candidate. Its stability over the cosmological time scale is guaranteed by the $H$ parity under which only the Higgs boson has negative parity at low energy. The triple vertices of $H W W, H Z Z$ and $H \bar{f} f$ vanish. However the 4-point vertices of $H H W W, H H Z Z$, and $H H f \bar{f}$ are allowed.

Interpreting the WMAP data on the relic density as not overclosing the Universe, the Higgs boson mass is constrained from below as $m_{H} \geq 70 \mathrm{GeV}$. Noting that the heavy Higgs boson generates more efficient annihilation into $W^{+} W^{-}$and $Z Z$, we study the cosmic ray positron and antiproton spectra and compare them with the PAMELA and Fermi/LAT observations. We do not aim at explaining the up-rising positron spectrum observed with the PAMELA. Instead we use the data to constrain the model. The Higgs boson annihilation rate is shown to increase with increasing Higgs boson mass. The $m_{H}=82 \mathrm{GeV}$ is marginally consistent with both the observed positron and antiproton spectra. However, the Higgs boson mass of $90 \mathrm{GeV}$ or more is obviously ruled out by the observed data.

Our purpose is to constrain the $S O(5) \times U(1)$ gauge-Higgs unification model by the PAMELA and Fermi/LAT data, which turned out to be very significant. The present limit on the model comes from the unitarity requirement, which limits the Higgs boson to be

less than about $\mathcal{O}(1) \mathrm{TeV}$ [21]. We show explicitly in this paper that the Higgs boson mass cannot be larger than $90 \mathrm{GeV}$, otherwise it overruns the observed positron and antiproton spectra of PAMELA and Fermi/LAT. Together with the constraint on the closure of the Universe, the gauge-Higgs boson mass as CDM is now restricted to $70-90 \mathrm{GeV}$.

\section{Acknowledgments}

We thank Professor Hosotani for useful discussion on the $H$ parity. The work was supported in parts by the NSC of Taiwan under Grant Nos. 96-2628-M-007-002-MY3 and the 
WCU program through the KOSEF funded by the MEST (R31-2008-000-10057-0).

[1] B. W. Lee and S. Weinberg, Phys. Rev. Lett. 39, 165 (1977).

[2] J. Dunkley et al. [WMAP Collaboration], Astrophys. J. Suppl. 180, 306 (2009).

[3] O. Adriani et al. [PAMELA Collaboration], Nature 458, 607 (2009).

[4] O. Adriani et al., Phys. Rev. Lett. 102, 051101 (2009).

[5] A. A. Abdo et al. [The Fermi/LAT Collaboration], Phys. Rev. Lett. 102, 181101 (2009).

[6] F. Aharonian et al. [H.E.S.S. Collaboration], Astron. Astrophys. 508, 561 (2009).

[7] See e.g., L. Bergstrom, T. Bringmann and J. Edsjo, Phys. Rev. D 78, 103520 (2008); K. Ishiwata, S. Matsumoto and T. Moroi, Phys. Lett. B 675, 446 (2009); P. Grajek, G. Kane, D. Phalen, A. Pierce and S. Watson, Phys. Rev. D 79, 043506 (2009); J. H. Huh, J. E. Kim and B. Kyae, Phys. Rev. D 79, 063529 (2009);

[8] See e.g., D. Hooper and K. M. Zurek, Phys. Rev. D 79, 103529 (2009); Y. Bai and Z. Han, Phys. Rev. D 79 (2009) 095023; S. C. Park and J. Shu, Phys. Rev. D 79, 091702 (2009).

[9] See e.g., A. Ibarra and D. Tran, JCAP 0902, 021 (2009); P. J. Fox and E. Poppitz, Phys. Rev. D 79, 083528 (2009); Y. Nomura and J. Thaler, Phys. Rev. D 79, 075008 (2009); R. Harnik and G. D. Kribs, Phys. Rev. D 79, 095007 (2009); I. Cholis, D. P. Finkbeiner, L. Goodenough and N. Weiner, JCAP 0912, 007 (2009); D. Feldman, Z. Liu and P. Nath, Phys. Rev. D 79, 063509 (2009); C. R. Chen, F. Takahashi and T. T. Yanagida, Phys. Lett. B 671, 71 (2009); M. Cirelli, M. Kadastik, M. Raidal and A. Strumia, Nucl. Phys. B 813, 1 (2009); V. Barger, W. Y. Keung, D. Marfatia and G. Shaughnessy, Phys. Lett. B 672, 141 (2009); E. J. Chun and J. C. Park, JCAP 0902, 026 (2009).

[10] J. Diemand, M. Kuhlen, P. Madau, M. Zemp, B. Moore, D. Potter and J. Stadel, Nature 454, 735 (2008).

[11] M. Lattanzi and J. I. Silk, Phys. Rev. D 79, 083523 (2009); J. Bovy, Phys. Rev. D 79, 083539 (2009).

[12] N. Arkani-Hamed, D. P. Finkbeiner, T. R. Slatyer and N. Weiner, Phys. Rev. D 79, 015014 (2009).

[13] D. Malyshev, I. Cholis and J. Gelfand, Phys. Rev. D 80, 063005 (2009); D. Hooper, P. Blasi and P. D. Serpico, JCAP 0901, 025 (2009); H. Yuksel, M. D. Kistler and T. Stanev, Phys. 
Rev. Lett. 103, 051101 (2009); S. Profumo, arXiv:0812.4457 [astro-ph].

[14] N. J. Shaviv, E. Nakar and T. Piran, Phys. Rev. Lett. 103, 111302 (2009).

[15] J. R. Ellis, K. A. Olive, Y. Santoso and V. C. Spanos, Phys. Lett. B 565, 176 (2003).

[16] Y. Hosotani, K. Oda, T. Ohnuma and Y. Sakamura, Phys. Rev. D 78, 096002 (2008) [Erratumibid. D 79, $079902(2009)]$.

[17] Y. Hosotani and Y. Kobayashi, Phys. Lett. B 674, 192 (2009).

[18] Y. Hosotani, arXiv:1003.3129 [hep-ph].

[19] Y. Hosotani, P. Ko and M. Tanaka, Phys. Lett. B 680, 179 (2009).

[20] Y. Sakamura, Phys. Rev. D 76, 065002 (2007).

[21] N. Haba, Y. Sakamura and T. Yamashita, JHEP 1003, 069 (2010).

[22] K. Cheung and J. Song, Phys. Rev. D 81, 097703 (2010).

[23] A. W. Strong, I. V. Moskalenko, T. A. Porter, G. Johannesson, E. Orlando and S. W. Digel, arXiv:0907.0559 [astro-ph.HE].

[24] Y. Hosotani, Phys. Lett. B 126, 309 (1983); A. T. Davies and A. McLachlan, Phys. Lett. B 200, 305 (1988); Y. Hosotani, Annals Phys. 190, 233 (1989).

[25] S. Albino, B. A. Kniehl and G. Kramer, Nucl. Phys. B 725, 181 (2005). 